\title{
Nanostructures Properties Data System. Critical Evaluation Methodology
}

\author{
Aleks andr Ele tskii ${ }^{1,2}$, Adilbek Erkimbaev², Georgy Kobzev², Michael Trachtengerts ${ }^{2}$, \\ Vladimir Zitserman ${ }^{2, *}$ \\ ${ }^{1}$ Russian Research Centre "Kurchatov Institute”, Moscow, Russian Federation \\ ${ }^{2}$ Joint Institute for High Temperatures, Russian Academy of Sciences, Izhorskay a 13, Bldg. 2, Moscow, 125412, Russin Federation
}

\begin{abstract}
This paper reviews general problems appearing in collecting, categorization, and certification of numerical properties data for nanoscale objects. It is shown how their peculiar physical properties affect preparation of the data at the preliminary stage before moving to detailed data base design. As an example, the properties data of carbon nanoforms (nanotubes, graphene, etc) are presented. The key features of the nanosized data are revealed, such as: permanent variations of the properties nomenclature, dimensional effect, and high level of the data uncertainty. The procedure is proposed for data certification taking into account quantitative statement of uncertainty as well as quality indicators. The former present the completeness of the description both of an object and a method as well the result reproducibility.
\end{abstract}

Keywords Nanostructure, Nanomaterial, Numeric Properties Data, Dimensional Effect, Logical Structure Semistructured Data, Data Certification

\section{Introduction}

This paper focuses on building of nu merical databases on the properties of nanoscale objects. The main attention is paid to system of nanodata in general (per se) with its essential and specific features such as existing body of data, logical structure, format, representation in a data base (DB) etc. Variety of the synthesized nanoforms and types of objects with unique properties defined by dimensional factor makes impossible use of co mmon manner of handling when applied to numeric nanodata.

Here we summarize the general concepts and procedures mainly. Those were already taken as a sample at a DB building on carbon nanoforms - fullerenes, graphenes, nanocapsules, nanotubes, nanodiamonds, etc[1, 2]. All those were discovered during last decades, but till now there is no internationally adopted nomenclature and the general specification of the data. The state in this area dramatically differs from that with common materials.

On the other hand, large body of relevant data makes it promising today to choose nanocarbon as progenitor of suitable approach for evaluation properties data of the multitude of nanoscale objects. There is one more argument in support of drawing on nanocarbon data experience. We already have a lot of analytical works including reviews of

* Corresponding author:

vz1941@yahoo.com (Vladimir Zitserman)

Published online at http://journal.sapub.org/nn

Copyright (C 2012 Scientific \& Academic Publishing. All Rights Reserved one of the authors[3-7].

\section{Key Features of Nanostructures and Nanomaterials}

\subsection{Main Peculiarities of Numerical Data}

According to general definitions, nanoobjects are the objects that have less than $100 \mathrm{~nm}$ in any size (diameter, thickness, and the like). They are atomic and molecular clusters, grains, nanotubes, nanofibers, films, etc. Nanomaterials are bulk substances made from structural nanoscale units (for example, fullerite made from fullerenes). Even a cursory examination of properties of nanoobjects shows three main features which should be taken into consideration in efforts to compile and disseminate fully evaluated materials property data.

A large variety of existing object types can not be confined to fixed property nomenclature. Different kinds of objects have their own lists of important features that should be incorporated in a DB. They demand the development of a flexible logic structure capable to support such data.

Nanoobjects stay in intermediate position between single molecule and bulk substance. For this reason it is necessary to ascribe the nomenclature of macro properties to nanoscale objects. Examples are found in mechanical properties and thermal conductivity of carbon nanotube (CNT) and graphene, phase transitions in clusters [8], variations in the phase diagram diamond-graphite as one passes from bulk to nanoscale objects [9]. 
The properties show significant dependence on production methods (processing history, fabrication treatments etc). There are some sources of such uncertainty due to method and conditions of synthesis, as well as uncontrollable factors, such as defects in structures, impurity on surface, etc.

\subsection{Dimensional Effects}

The fundamental and universal reasons for deviations of numerical nanodata from related bulk properties lie in dependence of properties (structural, thermodynamic, electronic, transport etc.) from the characteristic size of a nanoobject. What is more, a distinction needs to be drawn between irregular dimensional dependence (with specific maxima in some cases) and regular (monotonous) inherent in the bulk objects. The first is irregular dependence of a property from number of particles that shows extremes at so-called "magic numbers", corresponding to the maximum of the cluster stability. Irregularities due to size of nanoobjects are observed also in mass spectra, ionization potentials and some other properties. The effect of size is the most principal reason of the data uncertainties. For example, thermal and electric conductivities of CNT depend significantly on its length. This is caused by a change of the transport mechanism (from ballistic to diffusive) at some CNT length[7]. Characteristic length, at which the change occurs, depends on concentration and type of defects that connected directly with processing history and conditions of specimen preparation.

As a result, the only CNT length is not sufficient to obtain complete characteristic. At least, it should be accompanied by data on synthesis method and processing history. The similar case is seen also for graphenes. Its transport properties are essentially dependent on lengthwise and crosswise extents of a specimen and also from edge structure (chirality). If reliable information on sizes, structure, chirality, and defects of an object is deficient or lacking in any manner, the data on properties have rather essential uncertainty. Thus, results of measurements [10] show that thermal and electric conductivities drop off by 2-3-order for individual single-walled CNT with increasing length less than $1 \%$.

Properties of multilayered CNT and graphenes depend appreciably on number of layers that also can be considered as manifestation of dimensional effect[11]. Thus, according to measurements [12], thermal conductivity of multilayered graphene decreases in inverse proportion to the number of layers $n$ and reaches crystal graphite value at $n>4$. In case of CNT the effect is opposite - thermal and electro conductivities of a specimen increase with enhancement of the number of layers [13].

In addition to dimensional effects, it is necessary to pay attention to uncertainty of the cross-section sizes. For example, measurements of thermal and electrical conductivities, elasticity modules etc., may be fulfilled if cross-section data of an object are available. If the thickness (width) of an object is one or several layers of atoms, choice of this pa- rameter (thickness or width) becomes arbitrary and brings forth additional problem.

This problem may be demonstrated with measure ments of thermal conductivity of graphene that defines as relation between heat flux through a sample and temperature gradient[14]. Obviously, the exact value of graphene layer thickness is required for calculation of the temperature gradient. It is commonly adopted that the distance between the nearest layers in the crystal graphite, equal 0.34 nanometers, is used. But sometimes, the characteristic size of carbon atom, that is less by a factor of $2-3$, is also used. As summary, arbitrary choice of the single-layer graphene thickness results in more than $100 \%$ uncertainty in the estimation of thermal conductivity.

The problem similar to the previous one appears at measurement of the Young modulus of CNT[6]. This property is defined as a relation between the stretching force and increasing sample length. In turn, the specific stretching coefficient is calculated from cross-section of a sample. There seemed no escaping the conclusion that uncertainty of CNT Young modulus can also reach $100 \%$.

The significant dependence of nanomaterial properties upon size of structural units means that a new parameter, size of a unit (crystalline particle, colloidal particle, etc), should be put into consideration. In many cases some subtle details, for example, the size distribution, volume ratio $\Delta V / V$ of space between grains, and so forth[15], may affect appreciably physical properties. Such supplementary data are also necessary for valid specification a nanomaterial, along with description of the material origin and its processing history. For example, full details are ultimately necessary for carbon cloth-like materials made with single-wall CNT, mu ltilayered graphene paper, CNT yarn, etc.

It is necessary to bear in mind that both geometrical and physical parameters of nanoscale units can show variations in values. Distribution of these parameters depends on methods and conditions in production and noticeably affects the numeric properties data. An example[16] demonstrates importance of detailed description. Processing of single-walled CNT film by nitric acid changes the type of electrical conductivity from semi-conductor to metallic[17]. Such processing removes attached molecules or absorbed radicals from surface of CNT that changes dramatically the electronic structure of an object.

The above example demonstrates once more that there are some other factors which have influence on data uncertainty - in particular molecules or radicals absorbed on surface. The physical properties of such objects are determined by a relatively large contribution of the surface as compared to bulk. Radicals' adsorption by the CNT or graphene surface is responsible for the variation of electronic structure that has an immediate impact on electrical properties. Thus, electrical conductivity of pure graphene sheet is 100-1000 times larger than that of partially oxidated graphene with $10 \%$ of oxygen[18]. It is caused by the energy gap that occurs at graphene oxidation. Thermal conductivity of graphene also decreases as the number of the absorbed 
radicals increases. The absorbed radicals act as the scattering centers for phonons, hindering collisionless movement along the specimen. There are some processes that remove radicals by heat or chemical treatment. To sum up, reliable data on type and amount of adsorbed radicals are necessary in addition to geometry and object structure characteristics for unambiguous characterization of the object as well as for data evaluation. Hence, the measured properties of nanoobjects have unremovable uncertainty that stems from their atomic structure. Nevertheless, needs of engineering design or scientific research demand, that the property data have a certain certification of quality or an integrated estimation of uncertainty. This estimation should be based on accessible data on size and structure of object, method of measurement, method of synthesis, etc. Some more details are considered in section 6 .

\subsection{Data Complexity}

In addition to high uncertainty of data, description of a nanoobject involves yet another peculiarity. The point is that properties make sense and value for users only when expanded descriptions of measurements method, state of the specimen, environmental and other conditions are available. Sufficient identification should include the whole set of the quantitative and qualitative features concerning structure, sizes, morphology, synthesis method, etc. It is pertinent to note that the same description is inherent not in objects of nanoworld only, but in materials elsewhere as well. Their properties are always defined by wide complex of factors (technology and structural features, environ ment, etc.). This feature differs distinctly a material from common substances which properties are defined by chemical composition and/or structural formula. In pursuing these aims the special concept of materials metrology[19] was designed to develop DB's on materials. The principles and practices set forth in this work demand that results of measurements were presented necessarily together with data on the measurement method and object characteristics, and reliability of numerical values is defined by scope of available data. In result of experience during development a DB for superconductors and ceramics, Munro[19] has suggested procedures for comprehensive certification of material quality data of any kind. The importance of these procedures increases at considering objects of nanoworld, as number of additional factors involved by synthesis and/or measurement methods, also increases. Any of these new factors have more effect on numeric data and formulations than it may be expected.

\section{The General Approach to Collecting and Processing of Nanoobject Data}

With use of data analysis principles [19-21] that are already applied at material science, and specific experience of data nanostructures evaluation[3-7], the approximate schematic on Figure 1 may be proposed. Design of data collec- tion is going on by two directions: the characterization of objects and the specification of properties.

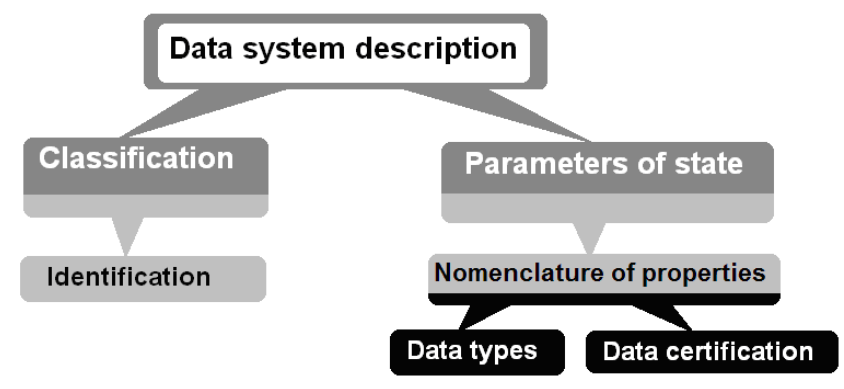

Figure 1. Schematic description of dat a system designing

The first of them (left line) based on a classification schema that allows the data to be organized or classified into categories in accordance with object's topology, sizes, etc. An expert in DB design needs to use an accepted scheme and must refer the object to one of its headings. Then a problem of its detailed identification is rising. For example, specific CNT can be identified with diameter, number of layers, chirality indexes, and, as appears from section 2, synthes is conditions. Generally, the set of identifying signs is specific for each of category and covers a rather large set of characteristics, such as the monomer formula, number of monomers in cluster or nanostructures, morphological features, thermal preh istory, external factors, etc. Identification procedures are discussed in detail in section 5.

The second line shown on Figure 1 (the right one) demonstrates the work steps applied at detailed elaboration of property data. The initial step involves choice of parameters of state and nomenclature of properties. Besides common temperature and pressure, data on structure and dispersion (the size distribution) as parameters may be taken for consideration. So the dilemma in characterization is appearing: whether to qualify the dispersion of an object (for instance, cluster) as a parameter of state or to take it as an identifying sign. The appropriate decisions should be made in data analysis for every object.

The nomenclature of properties depends essentially on the object class (headings of classification), and also on the specific purpose or functions to be served by data. It is essential that the nomenclature for clusters, nanotubes, and similar structures is extended to include both molecular and macroscopic properties as well. This feature shows the intermediate nature of nanostructures, being between molecule and bulk substance. For example, the whole set of the mechanical characteristics peculiar to engineering materials may be attributed to nanotubes also[6]. Another example adsorption properties that are mostly used as porous material characteristics [5].

When the nomenclature is accepted, the expert can start the main step - extraction of the relevant numerical values and formulas from publications and other sources. Specific features of an object and data presentation in original source dictate the type and format of data to be accepted in preparation for the data input. It is always preferable to store raw 
data for nanoobjects, which mainly presented in three basic forms (tabular, formulas, graphic), because they have exceptional variety of property values representations.

Regardless of adopted representation for numeric data, they are accompanied by some metadata in any information system[22]. This term (metadata) denotes data about the data, i.e. structured information that describes, explains, locates, or otherwise makes it easier to retrieve, use, or manage an information resource. When applied to DB on properties metadata point out names, designations, units of measure, measurement method (or that of evaluation) and presentation of property data and, more importantly, possible uncertainty. Data presentation, as a concept, is wide and permits different meanings that cover main details in property definition, type of data used, a set of measurements for the multidimensional data with lists of their types and values. Some elaboration may be critical to understanding or using property data, because property definition is connected frequently with context: a measurement method, model, scope of use, etc. Metadata permits linkage the property definition with the context; for instance, data must be accompanied by measurement methods, model, application and so on. The typical example is "hardness of material", a property defined by method of measurement (Knoop hardness, Vickers hardness, Rockwell hardness, etc.). Differences in definitions of thermodynamic properties relate to the accepted reference state, methods of numerical data representation (direct, difference from values at a reference state, ratio of the value to that at reference state, etc.), temperature scale. The metadata also follow data on uncertainties, as their representation have diverse kinds: absolute and relative values, confidence interval at a given level of confidence, etc. The last step finishes the data evaluation (section 6), i.e. it integrates numeric property data and metadata presented separately in their files.

\section{Categorization of Nanoobjects}

As a rule first step involves identification of an object (Figure 1) which sets apart it from the heading of clas sification according to adopted set of identifying signs. We have here analogy to identification in chemistry when a substance from a group (elements, oxides, hydrides, etc.) is pointed out by chemical formula.

No conventional classification of nanoobjects is yet accepted. For example, fullerenes and CNT may be named clusters and large molecules for both as well. So, fullerenes as molecules do not raise any doubt, but CNT, because of appreciable diversity in their size and structures, ambiguity in classification seems to be a cause of unavoidable difficulty. Problems arise also when we consider a family of graphenes. The classical definition of graphene corresponds to a single-layered hexagonal graphite structure. However, many authors consider the structures, consisting from two or even of several closed layers, as graphene as well. So, it is necessary to define and adopt the number of graphene layers when graphene converts to graphite. The question is typical for nanostructures with dimensional effects.

A simple scheme was suggested in[15], where authors divided the whole nanoworld on two kinds - separated individual nanoclusters and nanocluster's systems (materials). Moreover, they introduced 6 cluster types, based on methods of synthesis only: molecular ligands, gas ligandless, colloidal, solid-state, matrix, film. Thus, all kinds of fullerenes and CNTs come under the heading of ligandless gas phase clusters.

Pokropivny and Skorokhod[23] distinguish four types of objects by the dimensions as criterion, instead of separation of all nanostructures into two categories (materials and clusters). This number can have four values from 0 to 3 . Value $K=0$ means cluster with length no more than $100 \mathrm{~nm}$ in every dimension. On the contrary, value $K=3$ is applied to common macroscopic substance, or material. The prefix «nano» in this case only shows the size of elements that assemble the material. Intermediate values $K=1,2$ are applied to 1-dimensional and 2-dimensional structures, which have macroscopic size along one or two dimensions. For example, such are nanowires and nanofilms.

In the case, when criterion mentioned is used to certify structural elements that form the defined object, the dimension, however, can accept only 3 values $(L=0,1,2)$. Then the class of objects, that assembled by elements of same type, may be described by «nanoformula» K D L . All the clusters of $\mathrm{C}_{\mathrm{N}}$ type, that are chemical forms of carbon atoms, are referred to the sole 0D0 class, because dimension signs $(K=0, \quad \mathrm{~L}=0) \quad$ refer equally to cluster and monomer. On the other hand, nanotube or graphene assembled by those elements are defined by formulas 1D0 and 2D0. If an object is assembled by elements of several types, the formula assumes the form $\mathbf{K D}\{\mathbf{L}, \mathbf{M}, \mathbf{N} \ldots\}$ and $\mathbf{K} \geq \max \{\mathbf{L}, \mathbf{M}, \mathbf{N} \ldots\}$. The number of classes defined with this classification is essentially limited. For example, when only three structural elements are used, the number of classes is no more than 36[23].

\section{Object Identification}

Identification procedure sets apart unequivocally an object from a class of similar ones (CNT, graphenes, clusters, and etc.), that fall in the heading of the classification. For instance, it is necessary to use some identify ing signs of an object, such as chemical composition, size, structure, etc., to set apart it from the class, defined by topological nanoformula. Thus, the topological formula 0D0 for cluster $A_{N}$ should be followed by chemical formula of a monomer $A$, mono mer number $N$, and by symbol of point group $\left(\mathrm{D}_{3 \mathrm{~h}}, \mathrm{~T}_{\mathrm{d}}\right.$, $\mathrm{O}_{\mathrm{h}}$, etc). Precisely such tables of atomic and molecular clusters data were used for the Cambridge Cluster Database building (www-wales.ch.cam.ac.uk/CCD.html). Identification by number $N$ appears to be impractical when it became closer to $10^{3} \div 10^{4}$. In this case the more convenient characteristic is the linear size expressed in nanometers accompa- 
nied by crystal type and features of morphology.

The representation of modelling results for several carbon cluster families [24] may be an illustration for said above. The proper identification is achieved there by only pointing on cluster diameter and structure type: bucky-diamonds, icosahedral clusters, fullerenes, and fullerenelike structures (carbon cages and carbon onions). Analys is of nanodiamond detonation synthesis $[2,25]$ needs also to consider nanocluster types. Particles of nanographite, nanodiamond, and nanodiamond, covered with a graphite layer, appear in reaction zone. Each of them is qualified by diameter, as to the last type, it is characterized also by the layer thickness.

The other wide class of nanostructures is nanotubes. These objects are included in a class defined by the topological formula 1D0. Specific objects may be set apart from that class by monomer chemical formula $(\mathrm{C}, \mathrm{BN}, \mathrm{BeO})$, chirality indexes, diameter and number of walls. Besides, exact identification needs additional data on structural defects, state of surface, and some other factors induced by on material synthesis. These examp les show that the identification rules can not be set a priori, i.e. specific peculiarities of every class must be taken into account.

Table 1. AST M scheme for material identification[20]

\begin{tabular}{|c|c|}
\hline Number & Indicators \\
\hline 1 & Primary identifiers (e.g., material class) \\
\hline 2 & $\begin{array}{l}\text { Characterization of the material } \\
\text { (e.g., in terms of its chemical composition) }\end{array}$ \\
\hline 3 & $\begin{array}{l}\text { Widely recognized specification codes } \\
\text { (e.g., M-52 steel) }\end{array}$ \\
\hline 4 & Source of the material and its processing history \\
\hline 5 & $\begin{array}{l}\text { Geometrical details for the specimens used } \\
\text { in the measurements }\end{array}$ \\
\hline 6 & Any fabrication treatments (e.g., heat treatments) \\
\hline 7 & $\begin{array}{c}\text { Service history experienced by the specimen } \\
\text { used in the measurements }\end{array}$ \\
\hline
\end{tabular}

Identification of nanomaterials as macroscopic objects is more difficult than cases above, because it is necessary to elucidate a source material description without taking into account nanosized inclusions, and these inclusions separately as well. There is a general recommendation for identifying materials by American Society for Testing and Materials (ASTM) with seven distinct categories, see Table 1.

It is obvious, that identifiers for separate nanostructures can be applied also for nanosized inclusions. Some new parameters, such as volume or weight fraction of inclusions, dispersion, volume fraction of intergrain area and so forth[15] are necessary in the case. As a whole, nanostructure identification should meet two requirements: (1) use of extensive set of signs, defining size, chemical composition, structure, and other factors; (2) possibility to change the defining set while going from a nanostructure class to another one.

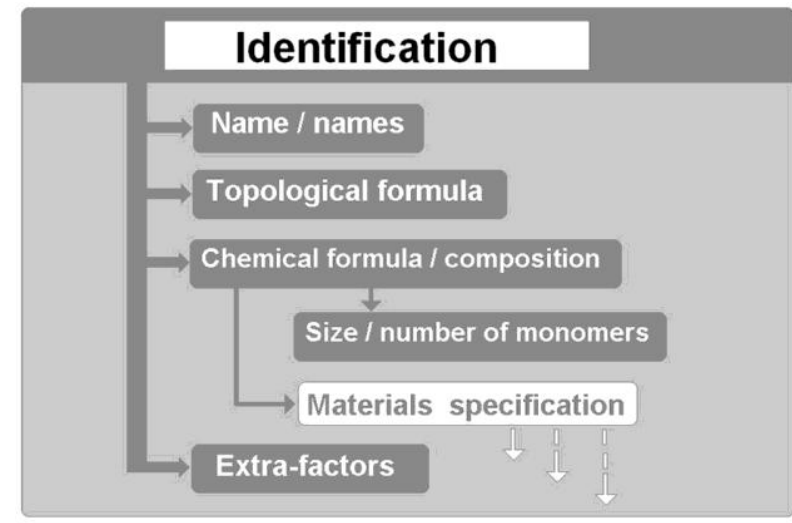

Figure 2. Schematic of the nanoscale objects characterization

Figures 2 and 3 present "frame", i.e. the exe mplary listing of data blocks that are intended for identification of objects. The scheme named as «Identification» displays necessity to consider supplementary identifiers for macroscopic objects (e.g. crystal symmetry data). The last of the blocks («extra-factors», Figure 3) refines data on structure, influence factors, synthesis, etc. for both object and particular specimen as well. Logical structure and type of the data in every block vary with nanostructure type. This is the foundation to implement context-dependent semistructured data concept $[22,26]$.

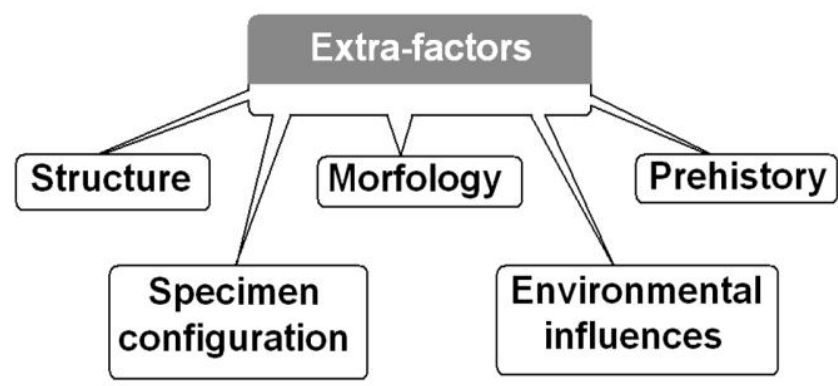

Figure 3. Data block "extra-fact ors"

\section{Data Certification}

\subsection{Procedures of the Data Certification}

Data certification (DC) is a set of procedures that fulfils multi-aspect evaluation of data presented and results in estimation of total uncertainty, i.e. error value and/or some data quality indicator. In some exceptional cases it may be enough to adopt a decision that the data are acceptable in accordance with some criteria.

According to the simplified scheme in Figure 4, the first step includes three procedures for evaluation of reliability, completeness, and consistency of the data. The first of them should show, whether identification (specification) of the object is completely presented in the data. This part of certification is important, as materials properties are of no value without detailed characteristics of the material. The 
identification may be called complete when the values of all identifying signs are known and the blocks, schematically outlined in Figure 2, are filled out.

The second DC procedure (Figure 4) should provide answer to the same question, relating now to measurement (or prediction) method, i.e. whether the description is sufficient for evaluation of results. Development of nanotechnologies was a result of widely used high-precision physical methods that allow determination of structural characteristics and chemical composition of a sample: electronic (ionic) microscopy; Raman-spectroscopy; methods of electronic spectroscopy (Auger-spectroscopy, X-ray photoelectronic spectroscopy, Electron Energy Loss Spectroscopy and many others. The foremost goal in describing measurement method is to present sufficient information for comparability and estimation of uncertainties. Sufficient information on applied method allows the estimation of reliability, taking into account, that all techniques have limited ranges of practical use.

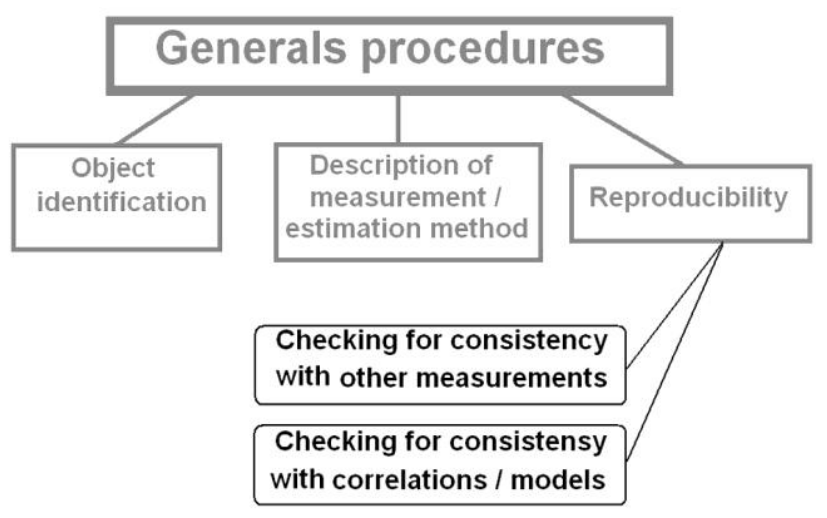

Figure 4. Schematic of the data certification

Correlations and theoretical methods must be characterized in the same manner with respect to assessment of reliability and completeness. As a rule, the universally accepted name of a method and its version, program realization, model parameters, and so on, are presented. For example, the investigation of carbon clusters energetics [24] was accompanied by detail description in such point: modelling is based on a self-consistent and environment-dependent (SCED) Hamiltonian, implemented in the framework of linear combination of atomic orbitals (LCAOs). As the method is a semiempirical one, the description includes the set of parameters, accepted in the work, so called optimized carbon parameters. The essential point for theoretical method in description and estimation of reliability is comparing results with available experimental data and/or alternative calculations.

The third procedure, shown in Figure 4, enables preliminary conclusion on reproducibility of measurements. The reproducibility is the mutual agreement among independent measurements, conducted and reported by the same or different laboratory. The higher level of confidence can be reached when validation of the value estimated by several predictive methods (quantum che mistry or semie mpirical) is possible.

When coupled with object and measurement method specification, the reproducibility allows us to provide ultimate assessment of the data. Qualitative (intuitive) characteristics may be discriminated by means of level quality indicators (high, middle, low), that is convenient in DB building. The corresponding metadata, that define the quality of data, include three indicators in this case: formally defined reproducibility of data as well as completeness of object and method specifications.

\subsection{Components of Nanoobject Data Uncertainty}

In addition to quality assessment, numerical estimation of the data uncertainty must be introduced. Some versions of estimation procedures for uncertainty are necessary according to variety of nanostructure types, forms, measurement methods, and, even when all objects are in nanocarbon family only. The first of them is applicable when data type depends only slightly on features peculiar to nanosize object. Thus, all publications on thermodynamic properties of fullerenes and fullerites represent results in the same form that is common in thermochemistry of traditional substances. Calorimetric methods together with standard estimations of molecular constants allow usage of common estimations of uncertainty based on any statistical method for treating [27]. The metadata must account for representation of uncertainty: standard deviation, level of confidence for the interval, combined uncertainty that includes extension of uncertainties from variables to the property. It is a common practice, when applied to nanostructures, to represent uncertainty as root-mean-square deviation expressed in absolute or relative values.

The second variant may be used when "ineradicable" uncertainty stems fro $m$ the dimensional effect or from specific synthesis method. The expert, responsible for data entry and maintenance of DB, takes into consideration possibilities of the method in combination with reproducibility of obtained results. As a result, the expert can offer an estimation of uncertainty in the form of value interval, but without probability interpretation, i.e. missing distribution law within the interval.

The third variant can be applied when theoretical methods of calculation are used. Notice that nanostructures data have a specific feature - published data, calculated by theoretical methods, become nowadays more numerous, along with improvement of data quality (accuracy and reliability). As appropriate estimation of uncertainty, some indicators may be considered, for example, (1) systematic error, inherent in each of methods, and usually presented in publication; (2) qualitative assessment of the reproducibility (measure of agreement) received from comparison with similar calculations or available experiments. The expert estimation presents the result in form of possible value interval as well as those pointed out in previous variants. 
Table 2. The categories proposed for property dat a of nanoobjects

\begin{tabular}{|c|c|c|}
\hline № & Dat a category & Assigning dat a to cat egories \\
\hline 1 & $\begin{array}{l}\text { Experimental, } \\
\text { high level of } \\
\text { reliability }\end{array}$ & $\begin{array}{c}\text { Availability of numerical estimations of } \\
\text { uncertainties at high level of quality } \\
\text { indicators }\end{array}$ \\
\hline 2 & $\begin{array}{l}\text { Experimental, } \\
\text { middle level of } \\
\text { reliability }\end{array}$ & $\begin{array}{c}\text { Availability of numerical estimations of } \\
\text { uncertainties at middle level at least one } \\
\text { of quality indicators }\end{array}$ \\
\hline 3 & $\begin{array}{l}\text { Experimental, } \\
\text { low level of } \\
\text { reliability }\end{array}$ & $\begin{array}{l}\text { Availability of numerical estimations } \\
\text { of uncertainties at low level at least } \\
\text { one of quality indicators. Absence of } \\
\text { numerical estimations of uncertainty }\end{array}$ \\
\hline 4 & $\begin{array}{l}\text { Theoretical (es- } \\
\text { timated), high } \\
\text { level of reliabili- } \\
\text { ty }\end{array}$ & $\begin{array}{l}\text { Availability of systematic error typical } \\
\text { of a given method at high level of quali- } \\
\text { ty indicators }\end{array}$ \\
\hline 5 & $\begin{array}{l}\text { Theoretical (es- } \\
\text { timated), mid- } \\
\text { dle level of relia- } \\
\text { bility }\end{array}$ & $\begin{array}{l}\text { Availability of systematic error typical } \\
\text { of a given method at middle level at } \\
\text { least one of quality indicators }\end{array}$ \\
\hline 6 & $\begin{array}{l}\text { Theoretical (es- } \\
\text { timated), the low } \\
\text { level of reliabili- } \\
\text { ty }\end{array}$ & $\begin{array}{l}\text { Availability of systematic error typical } \\
\text { of a given method at low level at least } \\
\text { one of quality indicators. } \\
\text { Unavailability of systematic error data }\end{array}$ \\
\hline 7 & Commercial & $\begin{array}{l}\text { Selected properties dat a, included in the } \\
\text { documentation given by the manufac- } \\
\text { turer or the supplier of a product }\end{array}$ \\
\hline 8 & Typical & $\begin{array}{l}\text { Typical values accepted from the litera- } \\
\text { ture, without indication on reliability } \\
\text { level. Provide order of magnitude esti- } \\
\text { mations or functional trends. Data } \\
\text { should be qualified as unevaluated }\end{array}$ \\
\hline
\end{tabular}

\subsection{Data Quality Categ ories}

Quality indicators, combined with numerical estimation of uncertainty, are a foundation for data to be distinguished by the categories, as it already was applied for common materials[19]. Table 2 shows how numeric data may be assigned to any of proposed categories defining their reliability. There are 8 such categories. Experimental as well as theoretical data may be assigned to be among three quality categories (1-3 for experimental, 4-6 for theoretical data). For instance, experimental data may be assign to the category 1, if statistical error is known (that is the common situation in research of macroscopic objects) and each indicator that define reproducibility and completeness of data is qualified with high level. In more difficult case (e.g., occurrence of dimensional effect) the same level of reliability is assigned when uncertainty is shown as interval of values and the same level of quality indicators is present.

Decrease in quality and/or absence of numerical estimation of uncertainty transfers the experimental data to cate- gory 2 or 3 . Theoretical data may be qualified in the same way - by availability of systematic error that is inherent in the model and quality indicators (categories 4-6).

In actual practice, some other categories are forced to be used, commercial and typical. Data of the first kind are provided by manufacturer to inform a customer on principal characteristics of products (for example, fullerene or CNT). Typical data are derived from preliminary reports with no indication of reliability or uncertainty. Nevertheless, these data may be used for order of magnitude estimates or functional dependencies. In the absence of alternatives commercial or typical data may be loaded into DB with indication that any assessment of reliability is not available.

\section{Conclusions}

The present work suggests general principles for data collection on properties of nanoobjects. They may be used as the guideline of data base building. The paper includes description of logical data structure, adapted to specific features of nanostructures, and methods of data certification and categorization.

When creating a logical structure an expert defines the necessary set of identifiers and nomenclature of properties, as well as specifies types and data formats for each attribute. The foreseen requirements for data structure, stemming from specific properties of nanostructures, can be met by the widely used semistructured data (SSD) model[22, 26]. The model has been introduced to accommodate all forms and kinds of data that come from multiple sources with differences in notation, meaning etc. In SSD model the information that is typically associated with a schema is contained within the data, which is sometimes called "self-describing". In such DB there is no clear separation between the data and the schema, and the degree to which it is structured depends on the application. The benefit is that these data do not have a rigidly and predefined schema.

Some different lines to attack building data base of that type were proposed based, for example, on XML tools [28]. We applied technology that used free distributed object-relation data base system with open code PostgreSQL. It combines traditional relational data model with maintenance of "fuzzy" data structure. The relative stability of the data "frame" described above (sections 4 and 5) serves as additional argument in supporting the technology inheriting the capabilities of conventional tools. The potential of PostgreSQL have appeared to be quite sufficient for DB on nanocarbon properties[1], despite exclusive variety of structures and materials of this class. Along with variety of data structures, there is a marked feature of numeric data for nanoobjects - their level of uncertainty is incomparably high. Some factors may be responsible for basically unavoidable uncertainty due to nanoscale nature of an object. An uncertainty also rises with involving into consideration both types - experimental and theoretical data in absence of re liable approach to assessment of confidence. 
So, it is possible to describe adequately confidence in data only by involving the complete body of available information on uncertainty, numeric assessments, as well as quality indicators. In the section 6 we developed the procedure that may be used for certification of data by introduction of some quality indicators and assigning data to categories of reliability (table 2 ). In a broad sense, reliability is defined by available numeric value of uncertainty, co mpleteness data on both object and research method, and reproducibility of measurements/estimations. The metadata include quality indicators (object, method, reproducibility) with estimation according to the three-level scale, category on reliability and the indexes defining the uncertainty (random or systematic, absolute or re lative, etc.).

The described conceptual scheme may be adjusted to arbitrary nanoscale objects by changing (or expanding) of classification scheme, identification standards, properties nomenclature, and, if it is necessary, the certification procedure. From a practical standpoint it is very important that the soft proposed here is fully suited to make such adjustment even an expert may lack sufficient knowledge of computer technology.

\section{ACKNOWLEDGEMENTS}

Authors thank the Russian Foundation for Basic Research for support (Grant No. 10-08-00623).

\section{REFERENCES}

[1] A.O. Erkimbaev, V.Yu. Zitserman, G.A. Kobzev, "Sy stematization of Data on the Physical and Chemical Properties and Application of Carbon Nanostructures", High Temperature, vol.48, no.6, pp.830-836, 2010.

[2] Y. Hu, O.A. Shenderova, D.W. Brenner, "Carbon Nanostructures: Morphologies and Properties", Journal of Computational and Theoretical Nanoscience, vol.4, no.2, pp.199-221, 2007.

[3] A.V. Eletskii, "Carbon nanotubes", Physics Uspehi, vol.40, no.9, 899-924, 1997.

[4] A.V. Eletskii, "Carbon nanotubes and their emission properties", Phy sics Uspehi, vol.45, no.4, pp.369-402, 2002.

[5] A.V. Eletskii, "Sorption properties of carbon nanostructures", Physics Uspehi, vol.47, no.11, pp.1119-1154, 2004.

[6] A.V. Eletskii, "Mechanical properties of carbon nanostructures and related materials", Physics Uspehi, vol.50, no.3, pp.225-261, 2007.

[7] A.V. Eletskii, "Transport properties of carbon nanotubes". Physics Uspehi, vol.52, no.3, pp.209-224, 2009.

[8] R.S. Berry, B.M. Smirnov, "Phase transitions in various kinds of clusters", Physics Uspehi, vol.52, no.9, pp.137-164, 2009.
[9] C.C. Yang, S. Li, "Size-Dependent Temperature-Pressure Phase Diagram of Carbon", J. Phy s. Chem. C, vol.112, no.5, pp.1423-1426, 2008.

[10] E. Brown, L. Hao, J.C. Gallop, J.C. Macfarlane, "Ballistic thermal and electrical conductance measurements on individual multiwall carbon nanotubes", Appl. Phys. Lett., vol.87, Ar\# 023107, 2005.

[11] A.V. Eletskii, I.M. Iskandarova, A.A. Knizhnik, D.N. Krasikov, "Graphene: fabrication methods and thermophysical properties", Physics Uspechi, vol.181, no.3, pp.233-268, 2011.

[12] S. Ghosh, W. Bao, D.L. Nika, S. Subrina, E.P. Pokatilov, C.N. Lau, A.A. Balandin, "Dimensional crossover of thermal transport in few-layer grapheme", Nature Materials, vol.9, no.7, pp.555-558, 2010.

[13] H.J. Li, W.G. Lu, J.J. Li, X.D. Bai, C.Z. Gu, "Multichannel Ballistic Transport in Multiwall Carbon Nanotubes", Phys. Rev. Lett., vol.95, Ar\#086601, 2005.

[14] D.L. Nika, E.P. Pokatilov, A.S. Askerov, A.A. Balandin, "Phonon thermal conduction in grapheme: Role of Umklapp and edge roughness scattering", Phys. Rev. B, vol.79, Ar\#155413, 2009.

[15] I.P. Suzdalev, P.I. Suzdalev, "Nanoclusters and nanocluster systems. Assembling, interactions and properties", Russian Chemical Reviews, vol.70, no.3, pp,177-210, 2001.

[16] Z.J. Han, K. Ostrikov, "Controlled electronic transport in single-walled carbon nanotube networks: Selecting electron hopping and chemical dopping mechanisms", Appl. Phys. Lett., vol.96, Ar\#233115, 2010.

[17] A.S. Lobach, L.I. Buravov, N.G. Spizyna, A.V. Eletskii, A.P Dementyev, K.I. Maslakov, "Temperture-dependent resistance of Single-Walled Carbon Nanotube Films", Khimiya Vys. Energiy, vol.45, no.4, pp.1-7, 2011.

[18] Y. Hernandez, V. Nicolosi, M. Lotya, et al, "High-yield production of grapheme by liquid-phase exfoliation of graphite", Nature Nanotechnology, vol.3, no.9, pp.563-568, 2008.

[19] R.G. Munro, "Data Evaluation, Theory and Practice for Materials Properties", Special Publication 960-11. Washington, DC: Materials Science and En gineering Laboratory, NIST, 2003.

[20] B. Moniz, "Nomenclature and current standards for identificaton of engineering materials". In: Newton, C.H. (Ed.) Manual on the Building of Materials Databases. ASTM Manual Series: MNL 19. Philadelphia: American Society for Testing and Materials, 1993.

[21] C.H. Newton, (Ed.) Manual on the Building of Materials Databases. ASTM Manual Series: MNL 19. Philadelphia: American Society for Testing and Materials, 1993.

[22] A.O. Erkimbaev, V.Yu. Zitserman, G.A. Kobzev, L.R. Fokin, "The logical structure of physicochemical data: Problems of numerical data standartization and exchange", Russian Journal of Phy sical Chemistry A, vol.82, no.1, pp.15-25, 2008.

[23] V.V. Pokropivny, V.V. Skorokhod, "New dimensionality classifications of nanostructures", Physica E., vol.40, no.7, pp.2521-2525, 2008). 
[24] M. Yu, I. Chaudhuri, C. Leahy, S.Y. Wu, C.S. Jay anthi, "Energetics, relative stabilities, and size-dependent properties of nanosized carbon clusters of different families: Fullerenes, bucky-diamond, icosahedral, and bulk-truncated structures", J. Chem. Phys., vol.130, Ar\#184708, 2009.

[25] G. Benedek, P. Milani, V.G., Ralchenko (Eds.), "Nanostructured carbon for advanced applications", NATO Science Series. II. Mathematics, Physics and Chemistry Vol. 24. Berlin, Heidelberg, Dordrecht, New York: Springer, 2001.
[26] S. Abiteboul, P. Buneman, S. Suciu, "Data on the Web: From Relations to Semistructured Data and XML", Burlington, Massachusetts: Morgan Kaufman Publishers, 2000.

[27] V.V. Diky, G.J. Kabo, "Thermodynamic properties of $\mathrm{C}_{60}$ and $\mathrm{C}_{70}$ fullerenes", Russian Chemical Reviews, vol.69, no.2, pp.95-104, 2000.

[28] M. Graves, Designing XML Databases., Jersey: Prentice Hall, 2002. 